\title{
The implications of processing event sequences for theories of analogical reasoning
}

\author{
SUSAN E. WHITELY and G. MICHAEL BARNES \\ University of Kansas, Lawrence, Kansas 66045
}

\begin{abstract}
Sternberg (1977a, 1977b) has proposed a componential theory of information processing on analogies. The current study attempts convergent validation of the basic findings in verbal analogies by a method that is based on different underlying assumptions. Although the data were generally consistent with Sternberg's theory, the data indicated that application is better described by two separate events. Furthermore, the extent of individual differences in strategy models was so substantial that a higher level processing operation, such as control strategies, should be postulated, rather than to support a single-strategy model as characterizing analogy solving.
\end{abstract}

Verbal analogies, such as the following,

Race:Man::Strain:?

(1) Science (2) Bacteria (3) Fatigue (4) Tension (Answer: Bacteria)

have increasingly become a target for cognitive research. Contemporary experimental research on verbal analogies has been primarily motivated by the presumed importance of analogical reasoning in intelligent behavior. That is, analogical reasoning is thought to be involved whenever an individual draws a parallel between different things in his experience. Research on verbal analogies is important, since it seeks to explain frequently occurring processes in thinking. Reitman's (1965) computer simulation of verbal analogy solving and Rumelhart and Abrahamson's (1973) semantic distance theory of analogical reasoning represent some major examples of the analogy research that has been generated from contemporary experimental research on cognition.

More recently, however, experimental research on verbal analogies has been guided by an added perspective: the need to explain individual differences in analogy solving. Verbal analogies have had a major role in the measurement of general intelligence, since they are the most heavily loading item on the general factor (i.e., Spearman's, 1927, "g") that is common among intelligence test items. Unfortunately, however, the nature of intelligence is not well understood because individual differences research has concerned trait organization rather than theoretical processes. Explaining performance on verbal analogies from individual differences in the underlying component events could potentially provide a more adequate foundation for a theory of intelligence.

This study was partially supported by General Research Fund 3472-X0-0038 to Susan E. Whitely, Principal Investigator, from the University of Kansas.
Recently, Sternberg (1977a, 1977b) has proposed a theory of analogical reasoning that not only is more general than previous work (Reitman, 1965; Rumelhart \& Abrahamson, 1973), but also explicitly considers individual differences. Sternberg's componential theory of analogical reasoning postulates six processing events: encoding, inference, mapping, application, justification, and preparation and response. Four alternative models are presented in which the processes are organized into strategies for solving the analogy. In all models, the processes are ordered into the same general sequence, but the way in which the processing events are executed (self-terminating vs. exhaustive mode) varies in models. Additionally, for the forced-choice analogies, such as ability test items, Sternberg presents two different strategies for scanning the alternatives. These strategies can be applied to any of the models, thus yielding eight models for forced-choice analogies.

Sternberg (1977a, 1977b) presents empirical evidence on the various processing models at both the group and individual subject levels. His major dependent variable is response time, which he attempts to reproduce from alternative mathematical models for the processing strategies. The parameters for the six processes in the mathematical models are estimated by regression, in conjunction with a priming method for presenting the analogy and measures of information differences between the analogy terms. Using a subtractive factor logic, it is assumed that the number of processing events occurring during the presentation of the full analogy problem can be controlled by varying the prime condition. Thus, processing durations for several events that occur in a specific sequence can be estimated separately. The prime is systematically varied so that some events occur prior to the presentation of the full problem. Several types of analogies-figural, verbal, and geometric-are modeled.

Although Sternberg (1977a, 1977b) interprets his data as supporting one of the models over the others, 
several inherent aspects of his methodology may limit his conclusions. As Garner, Hake, and Eriksen (1956) indicate, findings on covert processes may depend on the measuring operations. The limitations of Sternberg's methods will be described briefly here. A more detailed discussion is given in the Appendix.

First, the subtractive factor design requires some strong assumptions about the independence of priming and processing sequences. Not only must priming not influence subsequent processing, but the natural order of component event execution must correspond to the ' stimulus order given in the primes. Unfortunately, no data on the validity of this assumption are available.

Second, even given the validity of the design assumptions, some methodological problems may have limited the difference between self-terminating and exhaustive models of processing. Although all models reproduced analogy response times quite well (Models I-IV, on the average, accounted for $78 \%, 83 \%, 86 \%$, and $85 \%$ of the variance, respectively), the difference between the fully exhaustive Model I and the fully self-terminating Model IV is relatively small. However, the power to discriminate between the models may have been low for two reasons: measurement errors in the stimulus information indices, and problems in applying the priming method to self-terminating processes. With respect to the information indices, for verbal analogies the difference between the models depended on rated, rather than objective, measures of stimulus attributes. Errors in the ratings will lead to less discrimination between the models. With respect to the priming method, solution time estimates for processes occurring after the prime will be biased if the primed processing has not been exhaustive. If the primed processes are self-terminating, the remaining solution time partially reflects these processes, as well as the later processes in the sequence.

These methodological limitations indicate that Sternberg's results need convergent validation (Garner et al., 1956) by another method. The purpose of the current study is to test the componential theory and models by a method that is based on different underlying assumptions than subtractive factor modeling. Protocol analysis has been gaining popularity as a method for studying processing (e.g., Greeno, 1976; Simon, 1976). Although protocol analysis also requires strong assumptions, these assumptions are substantially different from those underlying subtractive factor models. One especially important difference is that protocol analysis does not require specifying an a priori sequence for the processing events. The current study uses protocol analysis on an analogy task to examine several aspects of componential theory: (1) the necessity of six component events (encoding, inference, mapping, application, justification, and preparation and response) to account for analogy solving, (2) the sequential nature of component event processes (i.e., the order of event execution and the exhaustiveness of the processing), and (3) the extent of individual variability in processing strategies.

\section{METHOD}

\section{Task Design}

The most direct technique for protocol analysis is for subjects to verbalize during problem solving. However, a pilot study indicated that this technique would not be successful for verbal analogies. Subjects of ten were unable to verbalize about the analogy solving process, and substantial eye movement between analogy stimuli was observed during periods of no verbalization. Apparently, the processing is performed too rapidly and automatically to be studied by introspective methods.

An analogy simulation task was devised as an alternative technique for protocol analysis. The major assumption underlying the simulation task is that processing sequences may be inferred from subjects' patterns of information requests about analogy stimuli. In the simulation task, the analogy stimuli consist of terms for which the subjects have no prior information relevant to the analogy problem. Thus, the subject must request information from the experimenter. Although the simulation task differs from standard verbal analogies in stimulus meaningfulness, it is still a fundamental analogy that requires the problem solver to draw parallels between stimuli from semantic information.

The stimuli in the analogy simulation task consisted of a multiple-choice analogy in the following structural form:

$$
\begin{gathered}
A: B:: C: \text { (Stem) } \\
D_{1} D_{2} D_{3} D_{4} \quad \text { (Alternatives) }
\end{gathered}
$$

The simulation task stimuli corresponding to this structural form are seven pronounceable nonwords, as follows:

$$
\text { Lyomon:Firmani::Duciver: ? (Stem) }
$$

Bansher Ponto Nax Squish (Altematives)

These seven stimuli refer to animals that could evolve on other planets. Figure 1 shows a picture of one of the animals used in the task introduction and its list of properties. The pictorial representations of the animals were adapted from a Smithsonian

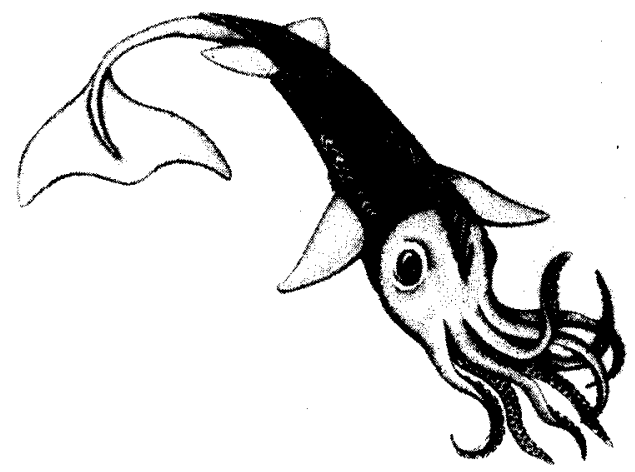

SQUISH

1. Lives on a high gravity world.

2. Does not migrate.

3. Is an aquatic predator.

4. Swims by jet propulsion with gills and tail.

5. Evolutionarily an old animal.

Figure 1. Example of a stimulus from the analogy simulation 
magazine feature on structurally feasible animals that, for some reason, did not emerge from the evolutionary process. An artist specialized in natural history illustrations was hired to produce drawings for the seven analogy animals and two animals to be used in the task instructions. The properties of the animals were coordinated with, but could not be directly inferred from, the animal's morphological structure. The properties concerned such things as habitat, locomotion, means of subsistence, migration, social structure, and so on.

As in standard analogies, only some of the information about the stimuli is relevant to the analogy. In the simulation task, the analogy is based on matching a predator-prey relationship between Duciver and Ponto to the predator-prey relationship between Lyomon and Firmani. Two associated properties were also relevant to this relationship: habitat and means of subsistence.

The analogy simulation task is administered individually, and the subject is given general instructions prior to examining two example stimuli. To prevent excessive rehearsal and thus increase memory load, subjects view the property list only while reading aloud. The experimenter scores each request for information as a separate step in the analogy solving process.

The major assumption of the task is that analogy processing sequences may be scored from information requests. The following scoring system was used: (1) Stimulus encoding is scored as the first information request for an analogy term; (2) inference and mapping are scored by sequential requests for $\mathrm{A}: \mathrm{B}$ or $\mathrm{A}: \mathrm{C}$, respectively; (3) application is scored by sequential requests for $C$ and at least two alternatives, $D_{1}$ and $\mathrm{D}_{2}$; and (4) a solution search is scored when at least two alternatives are viewed. A process is exhaustive if only one request for the appropriate stimuli is given during the entire protocol. Otherwise, the process is self-terminating.

In this scoring system, the attribute comparison processes, such as inference, mapping, and application are assumed to occur only during consecutive requests for information about the appropriate stimuli $(A: B, A: C$, and $A: D)$. Although it should be obvious that this assumption will never be fully met, the potential for covert processing leads to underestimating the degree of self-terminating processing. That is, the scoring system assumes exhaustive processing during stimulus presentation, and perhaps incorrectly, if no further information requests are given. Assuring that the assumption is at least reasonable depends partly on minimizing memory for the stimulus attributes so that relationships will not be constructed except during the relevant stimulus presentations. Reducing attribute memory by preventing rehearsal during information requests partly controls covert processing, but the size of the attribute list is another important memory factor that needs consideration.

The size of the attribute list for the final simulation task was determined from the results of a pilot study. The subjects were 38 undergraduates who were randomly assigned to one of two list size conditions: three vs. five attributes. Three dependent variables were calculated from the simulation task scores, total number of information requests, task solution, and number of relationship processes inferred from consecutive information requests. A multivariate test revealed significant differences among the conditions $[\mathrm{F}(3,34)=4.56, \mathrm{p}<.01]$. Individual $t$ tests revealed that, although probability of task solution did not vary between groups $(t=.33, p>.05)$, both number of requests and number of attribute comparisons were significantly greater in the five-attribute list condition $(t=2.93, p<.01$, and $t=2.81, p<.01$, respectively). Since the five-attribute list apparently decreases covert processing without increasing task difficulty, this condition was used in subsequent research with the simulation task.

\footnotetext{
Subjects

The subjects were 143 undergraduates at a large midwestern university. The students participated in the experiment to earn points in an introductory psychology course.
}

Materials

The materials consisted of the analogy simulation task as described above and 27 psychometric analogies from an item bank that had been developed for a college population (described in Tinsley \& Dawis, 1975; Whitely, 1977a).

\section{Procedure}

Subjects were first administered the 27 psychometric analogies under standard ability test instructions. The simulation task was then administered and scored as described above.

\section{RESULTS}

Some general descriptive statistics were calculated from the simulation task. The number of information requests (mean $=14.19, \mathrm{SD}=5.54$ ) was more than double the seven requests that would be sufficient to solve the analogy if all processing were exhaustive on initial encoding. Scoring of information requests during the initial encoding of stimuli revealed that $84.3 \%$ requested the stimuli in an order that corresponds to the processing event order of inference, mapping, and application; that is, the stem stimuli were requested in the order of A, B, C, followed by the alternatives. For $7.2 \%$, the stimulus order corresponded to the order of mapping, inference, and application; that is, the stem stimuli were requested in the order of A, C, B, followed by the alternatives. Of the remaining subjects, $6 \%$ requested one or more distractors prior to completely encoding the stem, while another $2.4 \%$ requested stem stimuli in idiosyncratic patterns (i.e., B, C, A; C, B, A).

After initial encoding of all stimuli, $58.7 \%$ of the subjects repeat a solution search by requesting information about two or more alternatives. However, only $19.7 \%$ of the solution searches after encoding involved all four alternatives. Apparently, some alternatives were falsified as possible solutions to the analogy during earlier processing. Another frequent pattern that was not directly anticipated from the componential theory was the request for a single alternative, preceded by one or more stem elements. Since this request involves no direct comparison between alternatives, it was tentatively labeled "confirmation." At least one confirmation was observed for $45.5 \%$ of the subjects.

Table 1 presents the frequencies and percentages for subjects using the various strategies on the simulation task. Strategy during encoding was scored separately from strategy after encoding because nearly $36 \%$ of the subjects requested repeated information about pairs of the stem elements prior to encoding the alternatives. Three separate patterns were observed, and a chi-square test revealed that the relative frequencies associated with these strategies were significantly different $\left(\chi^{2}=42.82\right.$, $\mathrm{p}<.01)$. The most frequent request was for the $A: B$ pair; the complete stem stimuli (A, B, and C) and the mapping pair $(\mathrm{A}: \mathrm{C})$ were requested far less frequently.

Substantial variability was also observed for strategies after complete encoding. Table 1 shows that only $18.2 \%$ of the subjects completed the problem immediately after encoding. This pattern would occur if all processing 
Table 1

Strategy Frequencies on the Analogy Simulation

\begin{tabular}{lrr}
\hline \multicolumn{1}{c}{ Variable } & N & $\begin{array}{r}\text { Per- } \\
\text { cent }\end{array}$ \\
\hline \multicolumn{1}{c}{ Strategy During Initial Encoding } \\
Total & & \\
Request encoding information only & 143 & 100.0 \\
Request for information comparisons & 92 & 64.3 \\
Inference comparison only (A:B) & 51 & 35.7 \\
Mapping comparison only (A:C) & 39 & 27.3 \\
Full stem comparison (A:B:C) & 5 & 3.5 \\
\multicolumn{1}{c}{ Strategy After Initial Encoding } & 7 & 4.9 \\
& & \\
Total & 143 & 100.0 \\
Immediate problem completion & 26 & 18.2 \\
Confirmation followed by problem completion & 33 & 23.1 \\
Distractor search before problem completion & 84 & 58.7 \\
Without stem return & 2 & 1.4 \\
Preceded by C request & 14 & 9.8 \\
Preceded by A:C request & 1 & .7 \\
Preceded by A:B request & 2 & 1.4 \\
Preceded by A:B::C request & 65 & 45.5 \\
\hline
\end{tabular}

were exhaustive, as in Sternberg's (1977a, 1977b) Model I. However, another $23.1 \%$ of the subjects made only one confirmation step before problem completion.

Table 1 also shows that for the $58.7 \%$ of the subjects who repeated solution searches after encoding, the most frequent pattern was to precede the search by requesting information about all stem elements. This is the pattèn that corresponds to Sternberg's (1977a, 1977b) Model IV, with all self-terminating processing. The second most frequent pattern for a solution search was to follow a request for only the $\mathrm{C}$ stimulus. This pattern corresponds to Sternberg's Model II, in which only application is self-terminating. Solution searches preceded by only $\mathrm{A}: \mathrm{B}$ or only $\mathrm{A}: \mathrm{C}$ (the latter corresponds to Model III) were rarely observed. The frequency differences between the patterns that correspond to the four models, with confirmation included in Model $\mathrm{I}$, were significant $\left(\chi^{2}=89.00, p<.01\right)$, but no difference was found between Model I with confirmation and Model IV $\left(\chi^{2}=.29, \mathrm{p}>.05\right)$. Thus, the exhaustive process model with confirmation provides a general model that is as good as the fully self-terminating model in accounting for the observed patterns.

Since the best single general model accounts for less than $50 \%$ of the cases, determining the number of strategy models needed to account for substantial numbers of cases is important. Figure 2 presents a summary of the cumulative percentage of cases that are accounted for under increasingly complex strategy models. It can be seen that adding the confirmation event to the least complex model, exhaustive processing, substantially increases the percentage of cases. Adding a model that permits self-terminating application gives less improvement than the addition of the confirmation event, but the improvement can be regarded significant, as the change is 2.39 times bigger than the standard error of the preceding percentages of cases. The next model adds mapping as self-terminating, and negligible improvement is shown. Finally, substantial improvement is gained by adding inference as self-terminating. These results show that four separate strategy models are needed to account for the observed patterns.

Table 2 presents a cross-classification of strategies executed consecutively. The strategies scored under the column categories are executed following the strategies scored under the row categories. The low-frequency categories of solution searches preceded only by mapping or inference were eliminated in this analysis. The 143 subjects executed a total of 219 strategy moves, giving a mean of 1.53. Significant association of the strategy types over times was indicated by a significant relationship among the cross-classified categories $\left(\chi^{2}=15.46, p<.05\right)$. The main contributors to the relationship are of the relative dependencies among confirmation followed by problem completion, and application followed by confirmation.

Strategies were also related to finding the correct solution to the analogy simulation task. A one-factor

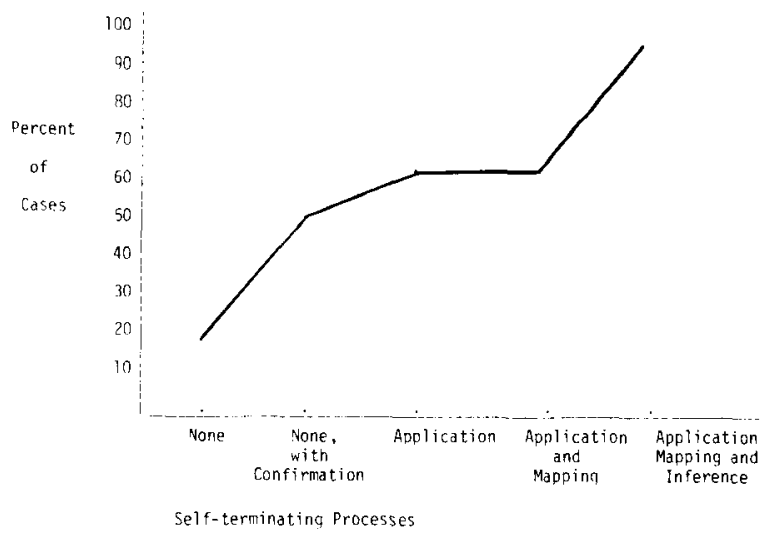

Figure 2. Percentage of cases accounted for by increasingly complex models.

Table 2

Probabilities and Frequencies of Strategy Sequences Over Time

Time $t+1$

\begin{tabular}{|c|c|c|c|c|c|c|c|c|c|c|}
\hline \multirow[b]{2}{*}{ Time $t$} & \multicolumn{2}{|c|}{$\begin{array}{c}\text { Problem } \\
\text { Completion } \\
\end{array}$} & \multicolumn{2}{|c|}{ Confirmation } & \multicolumn{2}{|c|}{ Application } & \multicolumn{2}{|c|}{$\begin{array}{l}\text { Inference, Mapping, } \\
\text { and Application }\end{array}$} & \multicolumn{2}{|c|}{ Total } \\
\hline & $\mathrm{N}$ & $\mathbf{P}$ & $\mathbf{N}$ & $\mathbf{P}$ & $\mathrm{N}$ & $\mathrm{P}$ & $\mathbf{N}$ & $\mathbf{P}$ & $\mathbf{N}$ & $\mathbf{P}$ \\
\hline $\begin{array}{l}\text { Confirmation } \\
\text { Application } \\
\text { Inference, Mapping, and Application }\end{array}$ & $\begin{array}{r}62 \\
9 \\
43\end{array}$ & $\begin{array}{l}.601 \\
.375 \\
.467\end{array}$ & $\begin{array}{l}22 \\
13 \\
20\end{array}$ & $\begin{array}{l}.214 \\
.542 \\
.217\end{array}$ & $\begin{array}{l}4 \\
0 \\
7\end{array}$ & $\begin{array}{l}.039 \\
.000 \\
.071\end{array}$ & $\begin{array}{r}15 \\
2 \\
22\end{array}$ & $\begin{array}{l}.146 \\
.083 \\
.239\end{array}$ & $\begin{array}{r}103 \\
24 \\
92\end{array}$ & $\begin{array}{l}.470 \\
.110 \\
.420\end{array}$ \\
\hline Total & 114 & .521 & 55 & .251 & 11 & .050 & 39 & .178 & 219 & \\
\hline
\end{tabular}


analysis of variance with four groups revealed that strategy during encoding, using the four categories in Table 1 , is significantly related to solving the task $(\mathrm{F}=3.72, \mathrm{p}<.01)$. The probabilities associated with analogy solving for the four strategy groups are as follows: encoding information only, .56; repeated inference stimuli, .75; repeated full stem stimuli, .63; repeated mapping stimuli, .17. Thus, obtaining additional inference information or the full stem information was associated with higher probabilities of problem solving, while obtaining mapping information was associated with a lower probability of problem solving.

The most complex strategy a subject executed after encoding was also related to solving the analogy. A one-factor analysis of variance indicated no significant differences in probabilities of solving the task $[F(3,139)=1.05, p>.05]$. The following probabilities of task solution were observed for four strategy categories: immediate problem completion, .50; confirmation followed by problem completion, .51; repeated application, .63; and repeated information on the stem, .66 .

The similarity of the simulation task to the ability test analogies was examined by comparing task outcomes. Task difficulties were quite comparable: Analogy solving probabilities of .53 and .52 , respectively, were obtained for the simulation task and the average ability test item. However, less comparability was observed for the correlation of response accuracy with total score on the analogy test items. Although solving the simulation task was significantly correlated with total score $(\mathrm{r}=.20$, $\mathrm{p}<.05$ ), it was not as discriminating as the psychometric analogies. The mean item/total score correlation in the analogy test was $.42(\mathrm{SD}=.16)$. If the simulation task item/total score correlation is located on this distribution, it is -1.5 standard score remits below the mean. Thus, the simulation task is comparable to a weak item in the analogy test.

\section{DISCUSSION}

The purpose of the current study was to test the componential theory by a converging operation. In this study, processing events were measured from a special analogy simulation task, using stimuli for which subjects had no prior information. The underlying assumption of the method is that processing events may be inferred from information-request patterns. Although the analogy simulation task differs from standard verbal inalogies in stimulus meaningfulness, it nevertheless zonstitutes a fundamental analogy that requires the oroblem solver to draw parallels between stimuli from iemantic information. The results from the simulation ask will be discussed with respect to the following zeneral issues in analogical reasoning: (1) the necessary somponents of analogical problem solving, (2) the equential nature of componential processes, and (3) the :xtent of individual differences in processing strategies.
Then, possible generalization to ability test analogies will be discussed.

\section{Necessary Components for Analogy Solving}

A major issue for analogical reasoning is the sufficiency and necessity of six component events to describe information processing in analogy solving. Sufficiency is difficult to assess from previous research using a subtractive factor approach because any unanticipated event will not be represented by either a unique prime or an information change measure. Several findings from the analogy simulation task are relevant to component sufficiency.

First, although the scoring categories constructed on the basis of six components were generally adequate to describe the protocols, one pattern that occurred in nearly half the protocols was not directly anticipated by the theory. This pattern, named confirmation, was a request for a single alternative, preceded by information relevant to inference, mapping, and application. Confirmation was most often a terminal event, as the data on strategy sequences indicated that the pattern was most frequently followed by problem completion. Although this could be considered a subset of Sternberg's (1977b) justification component, it may be preferable to consider this as a separate event. Justification was postulated as an optional component to test the validity of the preceding operations. Justification, however, is a complex event, because it can involve either testing a single response alternative for validity or discriminating between two or more options that are preceived to have equal validity. These two possibilities would seem to define rather different processing demands. Given the high frequency of the single-option pattern observed here, it may be meaningful to separate confirmation from the other types of justification.

Second, two separate findings, partial solution searches and self-terminating processing prior to encoding the alternatives, cannot be explained by Sternberg's (1977a, 1977b) single-stage application event. Although nearly all solution searches are preceded by requests for repeated information on inference, mapping, and application, only about $20 \%$ of the searches involve all four alternatives. Apparently, some alternatives are falsified before sufficient relational specificity is obtained to distinguish a unique solution. Thus, at least some relational information that was previously obtained was considered valid, rather than rejected when a unique solution was not found among the alternatives. The partial solution searches are consistent with a two-stage application event, image construction and response evaluation, to account for the apparent validity of some initial relational information. The repeated requests for relational information may be an attempt to obtain greater specificity of the image.

Similarly, self-terminating processing prior to 
encoding the alternatives supports an image construction phase that is separate from response evaluation. Nearly one-third of the subjects requested additional relational information prior to viewing the alternatives. Furthermore, subjects making this request had a higher likelihood of solving the analogy. Although this pattern could be an artifact of the task design, especially the memory load requirement, it is more likely that repeated processing of the stimuli continued until the solution image reached some critical level of specificity, as these pattern differences were significantly related to solving the task.

Thus, the current study gives empirical support for application as a two-stage event on verbal analogies. On the surface, these findings conflict with Sternberg's (1977b) support for a single-stage application event. However, Sternberg may not have found support for the two-stage application event for two reasons. First, partial solution choices could not be observed because his multiple-choice analogies had only two response alternatives. In two-alternative analogies, partial solution searches are not possible, since eliminating one alternative implies accepting the other as the solution. Thus, partial solution searches are a new finding in the current study, using an analogy with four response alternatives. Second, Sternberg's (1977b, p. 193) more direct test for the two-stage application event, adding a second application parameter to the response time models, was conducted on only one type of analogy. These were very easy pictorial analogies, presented as a true-false task with a single response alternative. The failure to separate image construction from response evaluation may have resulted from the more unconscious processing that seems to be more involved on easy items than on difficult items (Sternberg \& Rifkin, in press).

A two-stage application event can be supported on more general theoretical grounds. First, other models of analogical reasoning have postulated the construction of an image of the solution prior to response evaluation (e.g., Rumeihart \& Abrahamson, 1973). Second, image construction is also consistent with a means-end analysis to problem solving (Newell \& Simon, 1972). A means strategy seeks to solve problems by reducing the difference between the current state and the goal state (problem solution). But, in order to evaluate between possible moves, the problem solver must have a procedure for deciding which strategy will transform the current state into the closest approximation of the goal state. For analogy problems, an image of the solution can facilitate the application component in two ways. First, the solver can begin to specify the attributes the solution must have and falsify some alternatives, thus reducing the size of working memory. Second, the best alternative can be determined by comparison to the image of the solution. Thus, the separation of image construction from response evaluation is consistent with other theories that have some empirical support.

\section{Sequential Nature of Component Processes}

The findings from the analogy simulation task are relevant to two issues about processing sequences: (1) the order of processing events during initial encoding, and (2) the mode of event execution, selfterminating vs. exhaustive processing. With respect to the first issue, the results from the current study give empirical support for the processing event order that was assumed in previous studies. Nearly $85 \%$ of the subjects request stimulus information in patterns that correspond to the event order of inference, mapping, and application. For a rather small percentage of subjects (7.2\%), mapping preceded inference, while $6 \%$ of the subjects viewed a solution alternative prior to encoding the complete stem information, possibly as an attempt to direct the inference and mapping operations. Thus, although the general trend of inference followed by mapping and application is quite strong, some weak individual differences are also indicated.

With respect to the mode of event execution, the results support self-terminating events as a needed addition to models of processing on verbal analogies. Although the simulation task underestimates selfterminating operations because covert processing is not completely controlled, nearly $60 \%$ of the subjects overtly engaged in some self-terminating operations, as indicated by repeated information on the relational stimuli. Thus, self-terminating processing for at least some events are supported by convergent operations: previous work using the priming method, and the current protocol analysis.

Somewhat in conflict with previous work, however, are the number of self-terminating events needed to account for the data. Previous work indicated support for mapping and application as self-terminating, but found inconsistent results for inference. The protocol analysis indicates that all relational events-inference, mapping, and application-are self-terminating, since a pattern of repeated requests for all stem information was by far the most frequent pattern for nonexhaustive processing.

The current study also confirms Stemberg's (1977a, p. 238) finding for verbal analogies that no single-strategy model was consistently better at the individual subject level. The single best model, fully self-terminating processing, accounted for only about $45 \%$ of the subjects. These findings, however, in conjunction with the previous data, strongly argue against postulating a single general strategy model for verbal analogies. Although the best strategy model is fully self-terminating processing, the exhaustive model, with confirmation, accounts for $41.3 \%$ of the subjects. The next section elaborates on the theoretical implications of rejecting a general strategy model.

\section{Individual Differences in Processing Strategies}

The current findings suggest that strategy models may be an important source of individual differences in 
processing on verbal analogies. Four separate processing models were needed to account for the data. Many subjects' protocols were adequately described by the exhaustive processing model; others engaged in selfterminating processing on one or more events.

Additionally, individual variability in processing sequences was also observed prior to complete information encoding. Although the findings give only weak indications of individual differences in the initial order of processing events, substantial variability was found in the amount of repeated processing prior to the initial encoding of the solution alternatives. Furthermore, these strategy differences were associated with varying likelihoods of solving the analogies.

These results suggest that a higher level of processing than the component events or a general strategy model are needed to explain the varying strategies used by individuals. That is, variables corresponding to control strategies in general information processing models should be part of an adequate theory of analogical reasoning. An associated issue that needs further research is the extent to which the strategy variability may be attributed to reliable differences between subjects vs. more situational factors.

An important issue for individual differences research is the possible generalization of these results to the analogies that are found on popular ability tests. Two aspects of the current study may limit generalization to psychometric analogies. First, the measurement of processing sequences from the simulation task may have resulted in more conscious processing than is performed on standard analogies. Some recent evidence (Sternberg \& Rifkin, in press) suggests that conscious processing may change the algorithm for solving analogies. Second, the simulation task represents only one of eight types of analogies that have been identified on ability tests (Whitely, 1977b). Since these types depend on somewhat different cognitive abilities (Whitely, 1976), the simulation task may not fully represent the processing performed on ability test analogies.

Given these differences, at least some support for generalization to psychometric analogies can be given. The current study found that the simulation task difficulty was comparable to a psychometric analogy and that it had a significant, but low, correlation with ability test scores. A more recent study (Whitely \& Curtright, Note 1) found that the simulation task was correlated as highly with ability test scores as an average psychometric analogy.

In summary, although the results from the current study are based on a methodological operation that shares few common features with other studics on analogical reasoning, many of the findings about information processing on verbal analogies converge. Six component events were generally adequate to describe processing patterns, the inclusion of selfterminating modes of processing was supported, and substantial variability at the individual level was replicated. Furthermore, one major assumption of previous studies, that the order of component event execution is inference, mapping, and application, was given empirical support. However, in contrast to previous research, the current study indicates that the application event should be separated into two components, image construction and response evaluation, and that a new component event, confirmation, should be added. Furthermore, the results suggested that the extent of variability in strategy models was so substantial that a higher level processing operation, such as control strategies, should be postulated, rather than support a single-strategy model as characterizing analogy processing.

\section{REFERENCE NOTE}

1. Whitely, S. E., \& Curtright, C. The role of problem representation in solving verbal analogies. Unpublished manuscript, University of Kansas, 1979.

\section{REFERENCES}

Garner, W. R., Hake, H. W., \& Eriksen, C. W. Operationalism and the concept of perception. Psychological Review, $1956,63,149-159$.

Greeno, J. G. Indefinite goals in well-structured problems. Psychological Review, 1976, 83, 479-491.

Newelt, A., \& Simon, H. Human problem solving. Englewood Cliff, N.J: Prentice-Hall, 1972.

Re itman, W. Cognition and thought. New York: Wiley, 1965.

Rumelhart, P. E., \& Abrahamson, A. A. A model for analogical reasoning. Cognitive Psychology, 1973, 5, 1-28.

SimON, H. A. Identifying basic abilities underlying intelligent performance of complex tasks. In L. B. Resnick, (Ed.), The nature of intelligence. New York: Halsted Press, 1976.

Spearman, C. The abilities of man. New York: Macmillan, 1927.

Sternberg, R. J. Component processes in analogical reasoning. Psychological Review, 1977, 31, 356-378. (a)

STE RNBERG, R. J. Intelligence, information-processing and analogical reasoning: The componential analysis of human abilities. Hillsdale, N.J: Erlbaum, 1977. (b)

Sternberg, R. J., \& Rifkin, B. The development of analogical reasoning processes. Journal of Experimental Child Psychology, in press.

Tinsley, H. E. A., \& Dawis, R. V. An investigation of the Rasch simple logistic model. Sample free item and test calibration. Educational and Psychological Measurement, 1975, 35, 325-340.

WhITELY, S. E. Solving verbal analogies: Some cognitive components of intelligence test items. Journal of Educational Psychology, 1976, 68, 234-242.

Whitely, S. E. Information-processing on intelligence test items. Some response components. Applied Psychological Measurement, 1977, 1, 465-476. (a)

Whitely, S. E. Relationships in analogies: A semantic component of psychometric task. Educational and Psychological Measurement, 1977, 37, 725-739. (b)

\section{APPENDIX}

Componential Theory of Analogical Reasoning

This section is presented for the reader who wants greater detail on the componential theory and the modeling methods. Sternberg $(1977 \mathrm{a}, 1977 \mathrm{~b})$ postulates six processes for 
ENCODING

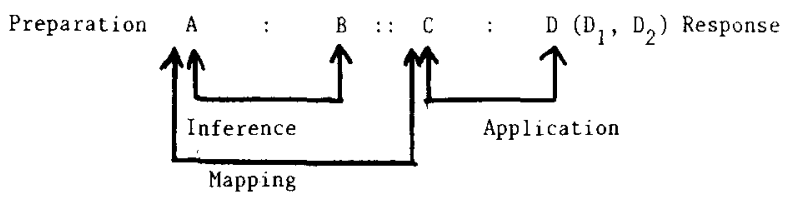

Figure A.

analogy solving. Figure A presents a schematic of these processes, for an analogy of the form $\mathrm{A}: \mathrm{B}:: \mathrm{C}: D$, such as in the following example:

Washington: $1:$ Lincoln: ?

$$
\text { (1) } 10 \text { (2) } 5 \text { (Answer = 5) }
$$

Encoding is the process by which the subject converts the physical stimulus term into an internal representation in working memory. In part, the internal representation consists of a list of attributes and properties of the stimulus that is activated from long-term memory during encoding. Inference involves a comparison between Stimuli A and B to discover the relationship between them. Mapping consists of discovering the rule between $A$ and $C$, and application generates the rule to find the ideal solution, $D$, and evaluate $D_{1}$ and $D_{2}$ for correspondence to $D$. The preparation and response components are control strategies for preparing to solve the analogy and translating the solution into a response. These are actually two processes, but Sternberg $(1977 \mathrm{a}, 1977 \mathrm{~b})$ generally combines them in his models. Justification (not shown in Figure A) is a process that occurs when a unique answer is not given by the sequence of events described above (i.e., $D_{1}$ and $D_{2}$ appear equally correct). In this stage, the relationships and encoding may be checked so that one answer can be justified as correct. Actually, as Sternberg describes justification, the process refers to several processing events that can possibly occur following failure to find an alternative that corresponds to the "ideal" answer.

In the analogy example given above, inference involves discovering that Washington's portrait is on a $\$ 1$ bill, whereas mapping involves discovering that both Washington and Lincoln are presidents with portraits on money. Application consists of specifying that Lincoln's portrait is on a $\$ 5$ bill and evaluating 10 and 5 as possible answers. The response phase gives 5 as the solution to the analogy.

In addition to the six processing events, two strategies for scanning the alternatives are given by Sternberg (1977a, 1977b). Alternating option scanning, a single attribute of the ideal answer $D$, is compared to all alternatives (i.e., $D_{1}$ and $D_{2}$ ) before checking a second attribute, and so on. In contrast, sequential option scanning involves checking all attributes of $\mathrm{D}$ against $D_{1}$ before evaluating $D_{2}$, and so on. For both strategies, the subject attempts to falsify alternatives so that an unfalsified alternative can be given as the correct answer.

Sternberg (1977a, 1977b) gives flowcharts for four strategy models for the basic processing events. Three of the eventsinference, mapping, and application-vary in the models. In Model I, the events are executed sequentially and all processing is exhaustive. In Model II, inference and mapping are exhaustive, but application is self-terminating. That is, application consists of only as many attribute relationships as are needed to give a unique answer. In contrast, exhaustive application consists of checking all attribute relationships. In Model III, inference is exhaustive, while mapping and application are self-terminating. In Model IV, inference, mapping, and application are all selfterminating. Sternberg (1977a) shows how the four models may be combined with the two alternative scanning strategies to yield eight possible models for forced-choice analogies.

\section{Supporting Data and Methods}

Table A presents the mathematical models corresponding to the four strategy models. For simplicity, the models presented here are for true-false analogies. Forced-choice analogy models are merely elaborations on the basic model for true-false analogies, as they specify how the alternatives are scanned. For each model, equations are given for the analogy solution time, under four priming conditions. When no priming occurs, all processing must occur during the presentation of the full analogy. As the cues presented during priming increase, the processing events occurring during the full analogy presentation are assumed to decrease. Also shown in Table $A$ are information measures, $f, g$, and $h$, that are used in the models. These measures are scored from the information changes between analogy terms to indicate the number of times a given processing event occurs for a given analogy item.

In Model I, under zero cues, no priming precedes the presentation of the full analogy, while for one cue, the A term is presented prior to the full analogy. The solution time difference $\left(\mathrm{ST}_{1}-\mathrm{ST}_{0}\right)$ between zero and one cue is the encoding of the $\mathrm{A}$ term, and this is represented by one less encoding Parameter a in the model for one cue. In the two-cue prime, both $A$ and $B$ are presented. Here, it is assumed that two terms are encoded, and the $A: B$ relationship is inferred during the prime. Thus, the solution time for two cues does not include these parameters. Under the three-cue prime, A, B, and C are presented, and it is assumed that encoding of three terms, inference, and mapping all occur during the prime. The remaining processing, encoding of one term and application, occurs during the full analogy presentation.

The assumptions underlying priming for inferences about processing events and the estimation of processing durations can now be examined more critically. First, if the processing events do not occur in the specified order (e.g., inference, mapping, and application), the parameter estimates will not reflect the duration of these processes in unprimed analogies. Second, the processing estimates will not be representative of what occurs on unprimed analogies if priming itself changes subsequent processing.

Also presented in Table A are mathematical models for the

Table A

Basic Equations for the Models of Analogical Reasoning

\begin{tabular}{|c|c|c|c|c|c|c|c|c|}
\hline Cues & Mod & lel I & & del II & & del III & Mod & del IV \\
\hline $\begin{array}{l}0 \\
1 \\
2 \\
3\end{array}$ & $\begin{array}{l}\mathrm{ST}_{0}=4 \mathrm{a}+\mathrm{fx} \\
\mathrm{ST}_{1}=3 \mathrm{a}+\mathrm{fx} \\
\mathrm{ST}_{2}=2 \mathrm{a}+ \\
\mathrm{ST}_{3}=\mathrm{a}+\end{array}$ & $\begin{array}{l}+g y+f z+c \\
+g y+f z+c \\
+g y+f z+c \\
+\quad+f z+c\end{array}$ & $\begin{array}{l}\mathrm{ST}_{0}=4 \mathrm{a}+\mathrm{f} \\
\mathrm{ST}_{1}=3 \mathrm{a}+\mathrm{f} \\
\mathrm{ST}_{2}=2 \mathrm{a}+ \\
\mathrm{ST}_{3}=\mathrm{a}+\end{array}$ & $\begin{array}{l}+g y+f^{\prime} z^{\prime}+c \\
+g y+f^{\prime} z^{\prime}+c \\
+g y+f^{\prime} z^{\prime}+c \\
+\quad+f^{\prime} z^{\prime}+c\end{array}$ & $\begin{array}{l}\mathrm{ST}_{0}=4 \mathrm{a}+\mathrm{f} \\
\mathrm{ST}_{1}=3 \mathrm{a}+\mathrm{f} \\
\mathrm{ST}_{2}=2 \mathrm{a}+ \\
\mathrm{ST}_{3}=\mathrm{a}+\end{array}$ & $\begin{array}{l}+g^{\prime} y^{\prime}+f^{\prime} z^{\prime}+c \\
+g^{\prime} y^{\prime}+f^{\prime} z^{\prime}+c \\
+g^{\prime} y^{\prime}+f^{\prime} z^{\prime}+c \\
+\quad+f^{\prime} z^{\prime}+c\end{array}$ & $\begin{array}{l}\mathrm{ST}_{0}=4 \mathrm{a}+\mathrm{f}^{\prime} \mathrm{x} \\
\mathrm{ST}_{1}=3 \mathrm{a}+\mathrm{f}^{\prime} \mathrm{x} \\
\mathrm{ST}_{2}=2 \mathrm{a}+ \\
\mathrm{ST}_{3}=\mathrm{a}+\end{array}$ & $\begin{array}{l}+g^{\prime} y^{\prime}+f^{\prime} z^{\prime}+c \\
+g^{\prime} y^{\prime}+f^{\prime} z^{\prime}+c \\
+g^{\prime} y^{\prime}+f^{\prime} z^{\prime}+c \\
+\quad+f^{\prime} z^{\prime}+c\end{array}$ \\
\hline
\end{tabular}

Note $-S T_{i}=$ solution time $i ; a=$ exhaustive figure scanning and encoding time; $x=$ exhaustive inference time; $y=$ exhaustive mapping time; $z=$ exhaustive application time; $x^{\prime}=$ self-terminating inference time; $y^{\prime}=$ self-terminating mapping time; $z^{\prime}=$ self-terminating application time; $c=$ constant preparation and response time; $N=$ total number of features; $T=$ truth index $(0=$ true, $l=$ false); $f=$ number of features changed from $A$ to $B ; g=$ number of features changed from $A$ to $C ; h=$ number of $D$ features correctly matched to $D^{\prime} ; f^{\prime}=[(N+T) /(N-h+1)] \times(f) /(N) ; g^{\prime}=[(N+T) /(N-h+1)] \times(g) /(N)$. 
self-terminating strategies. Under Model III, for example, mapping and application are both self-terminating. That is, they are performed only until a unique solution is found for the problem. For this model, the difference between the two-cue and three-cue solution times is the self-terminating mapping time, which is assumed to occur during the prime. However, this assumption seems unwarranted, as the subject cannot know prior to viewing the alternatives how many attributes need to be tested. Thus, if mapping is self-terminating, only one attribute relationship will be mapped during the prime, with the needed additional mapping occurring during the full analogy. In this case, the other parameters for the three-cue solution time are overestimated. The situation becomes even more critical for Model IV, as both self-terminating inference and mapping are assumed to occur during the prime. Thus, it is clear that priming can lead to biased estimates of self-terminating process durations in these models.

Another important feature of the mathematical models is the estimation of information changes. It can be seen in Table A that the only differences between the self-terminating and the exhaustive mathematical models are the estimates of information changes for each analogy. Specifically, the different estimates depend on the information difference between the specified alternatives, $D^{\prime}$ s, and the ideal answer, D. Although Sternberg (1977a, 1977b) was able to obtain an objective index of information changes for figural analogies, only subjective ratings of relational distance were available for verbal and geometric analogies. Thus, the difference in validity between self-terminating and exhaustive models is dependent on the validity of the ratings.

Measuring information change is especially critical for forcedchoice analogies, since the difference between the two models for scanning the alternatives, as well as the estimation of the justification process parameter, depends on the information change measure. The only forced-choice data given by Sternberg (1977a) are the geometric analogies, and rated measures of information change are used. Thus, any inadequacies in the ratings are directly reflected in the test between the two alternative scanning models and the estimation of the justification parameter.

(Received for publication January 30, 1979; revision accepted April 18, 1979.) 